INSTITUT NATIONAL DE RECHERCHE EN INFORMATIQUE ET EN AUTOMATIQUE

\title{
Optimal Control of the Atmospheric Reentry of a Space Shuttle by an Homotopy Method
}

Audrey Hermant

$$
\mathbf{N}^{\circ} 6627
$$

September 2008

Thème NUM

\section{apport}

derecherche 



\title{
Optimal Control of the Atmospheric Reentry of a Space Shuttle by an Homotopy Method
}

\author{
Audrey Hermant: \\ Thème NUM - Systèmes numériques \\ Équipes-Projets Commands \\ Rapport de recherche $n^{\circ} 6627$ - September 2008 - 23 pages
}

\begin{abstract}
This paper deals with the optimal control problem of the atmospheric reentry of a space shuttle with a second-order state constraint on the thermal flux. We solve the problem using the shooting algorithm combined with an homotopy method which automatically determines the structure of the optimal trajectory (composed of one boundary arc and one touch point).
\end{abstract}

Key-words: Optimal control, State constraint, Shooting algorithm, Homotopy method, Atmospheric reentry.

* CMAP, École Polytechnique, INRIA Saclay Île-de-France, Route de Saclay, 91128 Palaiseau, France.

Centre de recherche INRIA Saclay - Île-de-France

Parc Orsay Université

4, rue Jacques Monod, 91893 ORSAY Cedex

Téléphone : +33172925900 


\section{Commande optimale de la rentrée atmosphérique d'une navette spatiale par une méthode d'homotopie}

Résumé : Dans cet article, on s'intéresse au problème de commande optimale de la rentrée atmosphérique d'une navette spatiale avec une contrainte sur l'état du second ordre sur le flux thermique. Le problème est résolu par l'algorithme de tir combiné à une méthode d'homotopie qui détermine automatiquement la structure de la trajectoire (composée d'un arc frontière et d'un point de contact isolé).

Mots-clés : Commande optimale, contrainte sur l'état, algorithme de tir, méthode d'homotopie, rentrée atmosphérique. 


\section{Introduction}

The optimal control of the atmospheric reentry of a space shuttle is a challenging optimal control problem. It has been studied by many authors and solved using either direct methods (nonlinear programming), see [3, 7, 13, or indirect methods (multiple shooting), see 9 15] 16. Shooting methods, based on the resolution of a two- (or multi-) points boundary value problem, are choice methods when a high precision is required to compute a reliable optimal trajectory. However, it is well known that due to a small domain of convergence, it may be difficult to initialize the unknown variables to make the shooting algorithm converge. Moreover, in presence of constraints, the a priori knowlegde of the structure of constraints is required. To overcome these difficulties, continuations or homotopy methods can be used (see [1]), starting the homotopy path from an easier problem, e.g. the unconstrained problem (i.e. the problem without the state constraint), and introducing the constraints progressively, see e.g. 2, 11.

In the course of homotopy, the structure of solutions may vary, as well as the dimension of the unknown vector of shooting parameters. At those points where the structure changes, classical continuation methods cannot be applied. Somehow heuristic rules (justified in practice) for updating the structure of the trajectory were given e.g. in 22. It is of interest to be able to describe those changes in the structure for general control problems. Such results have been obtained in [5] for first-order state constraints and in [12] for second-order state constraints, providing a solid basis for a new homotopy algorithm which automatically determines the structure of the constraint and initializes the associated shooting parameters. The user has only to provide an initial guess for the solution of the unconstrained problem.

Our aim in this work is to illustrate this algorithm on the problem of the atmospheric reentry of a space shuttle with a second-order state constraint on the thermal flux. We follow the model of Bonnard, Faubourg, Trélat 9]. In 9], the authors computed an approximate solution by assuming that the control is bang-bang on interior arcs. (As it can be seen on Figure 5(a) this approximation is justified.) This simplification allows the authors to deduce the structure of the trajectory using a geometric analysis (see [10, 8]) and to obtain the solution by a shooting method without introducing the adjoint variables. In the present paper, we are able to check that the control is continuous (see Lemma 8) and that the Legendre-Clebsch condition holds excepted at the final time. The full set of necessary conditions is solved by the shooting algorithm. To this end, the geometric analysis in [10] 8] is used to initialize the unconstrained problem. Then we apply the homotopy algorithm of [12] which successfully finds the structure of the optimal trajectory, composed of one boundary arc and one touch point.

The paper is organized as follows. In section 2 the optimal control framework and the homotopy algorithm are presented. In section 3] we recall the model of 9] and discuss the validity of the assumptions of the homotopy algorithm. In section 4 we detail the initialization of the unconstrained problem. Finally, in section 5 we present the resolution of the constrained problem and give some comments on the numerical implementation. 


\section{Homotopy algorithm}

\subsection{General framework}

We consider general optimal control problems with a scalar control and scalar state constraint of the following form:

$$
\min \int_{0}^{t_{f}} \ell(u(t), y(t)) \mathrm{d} t
$$

subject to

$$
\begin{aligned}
& \dot{y}(t)=f(u(t), y(t)) \quad \text { for a.a. } t \in\left[0, t_{f}\right], \\
& g(y(t)) \leq 0 \text { for all } t \in\left[0, t_{f}\right], \\
& \psi\left(y(0), y\left(t_{f}\right)\right)=0, \\
& u(t) \in U
\end{aligned}
$$

where the controls $u(\cdot)$ are measurable and bounded functions, $U$ is a closed and convex subset of $\mathbb{R}$ and $\ell: \mathbb{R} \times \mathbb{R}^{n} \rightarrow \mathbb{R}, f: \mathbb{R} \times \mathbb{R}^{n} \rightarrow \mathbb{R}^{n}, g: \mathbb{R}^{n} \rightarrow \mathbb{R}$, and $\psi: \mathbb{R}^{2 n} \rightarrow \mathbb{R}^{r}$ are smooth functions. The classical (resp. generalized) Hamiltonian $H: \mathbb{R} \times \mathbb{R}^{n} \times \mathbb{R}^{n *} \rightarrow \mathbb{R}$ (resp. $\left.\mathcal{H}: \mathbb{R} \times \mathbb{R} \times \mathbb{R}^{n} \times \mathbb{R}^{n *} \rightarrow \mathbb{R}\right)$ of problem $(\mathcal{P})$ are defined by

$$
\begin{aligned}
H(u, y, p) & :=\ell(u, y)+p f(u, y), \\
\mathcal{H}\left(p_{0} ; u, y, p\right) & :=p_{0} \ell(u, y)+p f(u, y) .
\end{aligned}
$$

The well-known optimality condition of problem $(\mathcal{P})$ is as follows.

Definition 1. We say that $(u, y)$ is a Pontryagin extremal, if there exists $p_{0} \in \mathbb{R}_{+}$, functions $p:\left[0, t_{f}\right] \rightarrow \mathbb{R}^{n *}$ and $\eta:\left[0, t_{f}\right] \rightarrow \mathbb{R}$ of bounded variation such that $\eta\left(t_{f}\right)=0$, and $\lambda \in \mathbb{R}^{r *}$, $\left(p_{0}, p, \eta, \lambda\right)$ not all zero, such that

$$
\begin{aligned}
\dot{y} & =f(u, y) \\
-\mathrm{d} p & =\mathcal{H}_{y}\left(p_{0} ; u, y, p\right) \mathrm{d} t+g_{y}(y) \mathrm{d} \eta \\
u(t) & \in \operatorname{argmin}_{w \in U} \mathcal{H}\left(p_{0} ; w, y, p\right) \\
0 & \geq g(y(t)), \quad \mathrm{d} \eta \geq 0, \quad \int_{0}^{T} g(y(t)) \mathrm{d} \eta(t)=0, \\
0 & =\psi\left(y(0), y\left(t_{f}\right)\right), \quad p(0)=-\lambda \psi_{y_{0}}\left(y(0), y\left(t_{f}\right)\right), \quad p\left(t_{f}\right)=\lambda \psi_{y_{t_{f}}}\left(y(0), y\left(t_{f}\right)\right) .
\end{aligned}
$$

Moreover, if the final time $t_{f}$ is free,

$$
\min _{w \in U} \mathcal{H}\left(p_{0} ; w, y\left(t_{f}\right), p\left(t_{f}\right)\right)=0 .
$$

Theorem 2. An optimal trajectory of $(\mathcal{P})$ is a Pontryagin extremal.

The contact set of the constraint is defined by $I(g(y)):=\left\{t \in\left[0, t_{f}\right]: g(y(t))=0\right\}$. We consider the following assumptions:

(A1) The functions $\ell, \psi$ and $f$ (resp. $g$ ) are $C^{3}$ (resp. $C^{4}$ ) with locally Lipschitz continuous third-order (resp. fourth-order) derivatives.

(A2) The control $u$ is continuous and (strengthened Legendre-Clebsch condition)

$$
\exists \alpha>0, \quad H_{u u}(u(t), y(t), p(t)) \geq \alpha \quad \text { for all } t \in\left[0, t_{f}\right] .
$$

(A3) The state constraint is a regular state constraint of second-order. This means that the first-order time derivative of the constraint defined by $g^{(1)}(u, y):=g_{y}(y) f(u, y)$ does not depend on the control variable, i.e. $g_{u}^{(1)}$ is identically zero, while the second-order time derivative defined by $g^{(2)}(u, y):=g_{y}^{(1)}(y) f(u, y)$ satisfies

$$
\exists \alpha, \sigma>0, \quad\left|g_{u}^{(2)}(u(t), y(t))\right| \geq \alpha \quad \text { for all t }: \operatorname{dist}\{t ; I(g(y))\}<\sigma .
$$




\subsection{Description of the homotopy algorithm}

In this section we present the homotopy algorithm. Throughout the section it is assumed that $U=\mathbb{R}$, i.e. the control is unconstrained. We start by some definitions.

The structure of a trajectory is the number and order of boundary arcs (time intervals $\left(\tau_{e n}, \tau_{\text {ex }}\right)$ where $\left.g(y(t))=0\right)$ and touch points (isolated times $\tau_{\text {to }}$ such that $\left.g\left(y\left(\tau_{\text {to }}\right)\right)=0\right)$. The left- and right endpoints of a boundary arc $\left(\tau_{e n}, \tau_{e x}\right)$ are called respectively entry and exit points. A touch point $\tau$ is said to be essential if $[\eta(\tau)]>0$ and reducible if $g^{(2)}(u(\tau), y(\tau))<0$.

The shooting algorithm Let us first recall the shooting algorithm for optimal control problems with a second-order state constraint (see [14, 6, [15]). The alternative multipliers used in the shooting algorithm are denoted by $p_{2}, \eta_{2}$ and we define the alternative Hamiltonian $\tilde{H}: \mathbb{R} \times \mathbb{R}^{n} \times \mathbb{R}^{n *} \times \mathbb{R} \rightarrow \mathbb{R}$ by

$$
\tilde{H}\left(u, y, p_{2}, \eta_{2}\right):=H\left(u, y, p_{2}\right)+\eta_{2} g^{(2)}(u, y)
$$

where $H$ is the classical Hamiltonian (6). Througout the section, it is assumed that assumptions (A1)-(A3) hold and that

(A4) The trajectory has finitely many boundary arcs and finitely many touch points and the state constraint is not active at initial and final times, i.e. $g(y(0))<0$ and $g\left(y\left(t_{f}\right)\right)<0$.

Moreover, we assume that the extremals are normal, i.e. $p_{0}=1$, and hence Pontryagin's minimum principle holds by substituting to the generalized Hamiltonian $\mathcal{H}$ the classical Hamiltonian $H$.

Remark 3. Under our assumptions, the Hamiltonian is constant along a Pontryagin extremal so that (13) is equivalent to, for any $t^{*} \in\left[0, t_{f}\right]$ (in particular $t^{*}=0$ )

$$
H\left(u\left(t^{*}\right), y\left(t^{*}\right), p\left(t^{*}\right)\right)=0 .
$$

Assume that the structure of the trajectory is known and given e.g. by the variable $\mathcal{S}:=\left[s_{1}, \ldots, s_{N}\right]$ where the size $N$ is variable, $s_{i} \in\{1,2\}$ for $i=1, \ldots, N$, and "1" stands for a touch point and "2" for a boundary arc. Then the dimension of the shooting function is given by $d_{\mathcal{S}}:=2 n+r+1+(2 n+2) \sum_{i=1}^{N} s_{i}$. More precisely, a vector of shooting parameters $\theta \in \mathbb{R}^{d_{\mathcal{S}}}$ and the shooting function $\mathcal{F}_{\mathcal{S}}: \mathbb{R}^{d_{\mathcal{S}}} \rightarrow \mathbb{R}^{d_{\mathcal{S}}}$ associated with the structure $\mathcal{S}$ are as follows:

$$
\theta:=\left(\begin{array}{c}
y^{0} \\
p^{0} \\
\lambda \\
t_{f} \\
a_{1} \\
\vdots \\
a_{N}
\end{array}\right), \quad \mathcal{F}_{\mathcal{S}}(\theta):=\left(\begin{array}{c}
p^{0}+\lambda \psi_{y_{0}}\left(y^{0}, y\left(t_{f}\right)\right) \\
p_{2}\left(t_{f}\right)-\lambda \psi_{y_{f}}\left(y^{0}, y\left(t_{f}\right)\right) \\
\psi\left(y^{0}, y\left(t_{f}\right)\right) \\
H(u(0), y(0), p(0)) \\
b_{1} \\
\vdots \\
b_{N}
\end{array}\right)
$$

where $\left(y^{0}, p^{0}\right)$ is the initial value of state and costate, $\lambda$ is the multiplier associated with the constraint (4), $t_{f}$ is the (free) final time, and, for $i=1, \ldots, N, a_{i}, b_{i} \in \mathbb{R}^{(2 n+2) s_{i}}$ are as follows. If $s_{i}=1$ (touch point $\tau_{t o}^{i}$ ):

$$
a_{i}=\left(\begin{array}{c}
\nu_{t o}^{i} \\
\tau_{t o}^{i} \\
y_{t o}^{i} \\
p_{t o}^{i}
\end{array}\right), \quad b_{i}=\left(\begin{array}{c}
g\left(y\left(\tau_{t o}^{i}\right)\right) \\
g^{(1)}\left(y\left(\tau_{t o}^{i}\right)\right) \\
y_{t o}^{i}-y\left(\tau_{t o}^{i}\right) \\
p_{t o}^{i}-p\left(\tau_{t o}^{i-}\right)+\nu_{t o}^{i} g_{y}\left(y\left(\tau_{t o}^{i}\right)\right)
\end{array}\right)
$$

$\mathrm{RR} \mathrm{n}^{\circ} 6627$ 
where $\nu_{t o}^{i}$ is the jump parameter of the costate and $\left(y_{t o}^{i}, p_{t o}^{i}\right)$ the state-costate value at touch point $\tau_{t o}^{i}$. If $s_{i}=2$ (boundary $\left.\operatorname{arc}\left(\tau_{e n}^{i}, \tau_{e x}^{i}\right)\right)$ :

$$
a_{i}=\left(\begin{array}{c}
\nu_{e n}^{1, i} \\
\nu_{e n}^{2, i} \\
\tau_{e n}^{i} \\
y_{e n}^{i} \\
p_{e n}^{i} \\
\tau_{e x}^{i} \\
y_{e x}^{i} \\
p_{e x}^{i}
\end{array}\right), \quad b_{i}=\left(\begin{array}{c}
g\left(y\left(\tau_{e n}^{i}\right)\right) \\
g^{(1)}\left(y\left(\tau_{e n}^{i}\right)\right) \\
g^{(2)}\left(u\left(\tau_{e n}^{i-}\right), y\left(\tau_{e n}^{i}\right)\right), \\
y_{e n}^{i}-y\left(\tau_{e n}^{i}\right) \\
p_{e n}^{i}-p\left(\tau_{e n}^{i-}\right)+\nu_{e n}^{1, i} g_{y}\left(y\left(\tau_{e n}^{i}\right)\right)+\nu_{e n}^{2, i} g_{y}^{(1)}\left(y\left(\tau_{e n}^{i}\right)\right) \\
g^{(2)}\left(u\left(\tau_{e x}^{i+}\right), y\left(\tau_{e x}^{i}\right)\right) \\
y_{e x}^{i}-y\left(\tau_{e x}^{i}\right) \\
p_{e x}^{i}-p\left(\tau_{e x}^{i}\right)
\end{array}\right) .
$$

where $\nu_{e n}^{1, i}, \nu_{e n}^{2, i}$ are the jump parameters of the costate at entry times, and $\left(y_{e n}^{i}, p_{e n}^{i}\right)$ and $\left(y_{e x}^{i}, p_{e x}^{i}\right)$ are the state-costate values at respectively entry and exit points. The trajectory and multipliers $\left(y, u, p_{2}, \eta_{2}\right)$ are the solution of, on the interior of each $\operatorname{arc}\left(\tau, \tau^{\prime}\right)$ (which is a boundary arc if $\left(\tau, \tau^{\prime}\right)=\left(\tau_{e n}^{i}, \tau_{e x}^{i}\right)$ and an interior arc if $\tau$ is one of $0, \tau_{t o}^{i}$, or $\tau_{e x}^{i}$ and $\tau^{\prime}$ is one of $\tau_{t o}^{i+1}, \tau_{e n}^{i+1}$, or $t_{f}$, for some $\left.i=1, \ldots, N\right)$ :

$$
\begin{aligned}
\dot{y} & =f(u, y), \\
\dot{p}_{2} & =-\tilde{H}_{y}\left(u, y, p_{2}, \eta_{2}\right), \\
0 & =\tilde{H}_{u}\left(u, y, p_{2}, \eta_{2}\right), \\
0 & =g^{(2)}(u, y) \quad \text { on boundary arcs, } \\
0 & =\eta_{2} \quad \text { on interior arcs, }
\end{aligned}
$$

with initial values of the state and costate $(y(\tau), p(\tau))$ given by $\left(y^{0}, p^{0}\right)$ if $\tau=0,\left(y_{t o}^{i}, p_{t o}^{i}\right)$ if $\tau=\tau_{t o}^{i},\left(y_{e n}^{i}, p_{e n}^{i}\right)$ if $\tau=\tau_{e n}^{i}$, and $\left(y_{e x}^{i}, p_{e x}^{i}\right)$ if $\tau=\tau_{e x}^{i}$. The boundary arcs are allowed to have a nonpositive length, i.e. $\tau_{e n}^{i} \geq \tau_{e x}^{i}$, in that case (16)-(17) are integrated backwards starting from $\tau_{e n}^{i}$ until $\tau_{e x}^{i}$ is reached. On interior arcs, $\eta_{2}=0$ by (20) and under assumption (A2) (since we have $p_{2}=p$ on interiors arcs, see [6]), (18) allows us to express $u$ as a smooth function of the state and costate $u=\Upsilon\left(y, p_{2}\right)$. On boundary arcs, by (A3) and (19) $u$ can be expressed as a smooth function of the state $u=\Upsilon_{b}(y)$ and we have $\eta_{2}=-H_{u}\left(u, y, p_{2}\right) / g_{u}^{(2)}(u, y)$ by (18).

The shooting algorithm consists in finding a zero of the shooting function by applying e.g. a Newton method. Under assumptions (A1)-(A4), a zero of the shooting mapping is associated with a normal Pontryagin extremal $\left(p_{0}=1\right)$ iff (see [6] Corollary 2.17] and [12] Remark 6.4])

$$
g(y(t)) \leq 0, \quad \ddot{\eta}_{2} \geq 0 \text { on boundary arcs, } \quad \nu_{t o}^{i} \geq 0 \text { for all touch points } \tau_{t o}^{i} .
$$

Remark 4. In order to gain numerical stability, we consider here a slightly different shooting function to that of [12] by adding to the set of shooting parameters the value of state and costate at junction times. We detail below the straightforward extension of the homotopy algorithm in this case.

Homotopy algorithm The principle of the homotopy algorithm is to solve, with the shooting algorithm, a sequence of problems with state constraint depending on a parameter $\mu \in[0,1]$

$$
g^{\mu}(y):=g(y)-(1-\mu) M \leq 0,
$$

where the constant $M>0$ is large enough so that the state constraint $g^{0}(y)$ is not active. For $\mu=1$ we recover our problem $(\mathcal{P})$. Starting with $\mu=0$ (unconstrained problem), $\mu$ is progressively increased until reaching $\mu=1$. Doing so the structure of solutions may vary. 
It has been shown in [12] that under the uniform strict complementarity assumption on boundary arcs below

$$
\exists \alpha>0, \quad \dot{\eta}(t) \geq \alpha \quad \text { on boundary arcs, }
$$

changes in the structure of the constraint may occur only in presence of a nonessential or a nonreducible touch point. In the neighborhood of a nonessential reducible touch point, the only two possibility for the state constraint are to be inactive or to be active at a single touch point. In the neighborhood of a nonreducible essential touch point $\tau$, it is shown in 12] that there exists a function $\Lambda(\tau)$ such that if $\Lambda(\tau)<0$, the only two possibilities for the state constraint are to be active at a single touch point or at a single boundary arc, the latter satisfying (23), and if $\Lambda(\tau)>0$, the only two possibilities for the state constraint are to be active at one or two touch points. (If $\Lambda(\tau)=0$, any structure is a priori possible in the neighborhood of a nonreducible touch point.)

It is assumed in the homotopy algorithm that $\Lambda(\tau)<0$ for all nonreducible touch points $\tau$, i.e. nonreducible touch points turn into boundary arcs. The case where $\Lambda(\tau)>0$ (transformation of a nonreducible touch point into two touch points) is more difficult to handle in the homotopy algorithm since the Jacobian of the shooting function becomes singular if the two touch points are not appropriately initialized (see [12]). The computation of this function $\Lambda(\tau)$ is, though possible, difficult in practice since it requires the computation of higher derivatives of the functions of the problem. However, we check all along the homotopy path that the strict complementarity assumption 23) holds (see Remark below), and hence, we check a posteriori that the assumption $\Lambda(\tau)<0$ is valid.

Remark 5. We are able to check numerically in the course of the homotopy algorithm that the strict complementarity assumption (23) is satisfied. The state constraint multiplier $\eta$ can be computed on the interior of boundary arcs by $\eta=\dot{\eta}_{2}+C s t$, where $\eta_{2}$ is the alternative multiplier of the shooting algorithm (see [6]), and we have by time differenciation of (18) (omitting arguments $\left(u, y, p_{2}, \eta_{2}\right)$ )

$$
\dot{\eta}_{2}=-\left(g_{u}^{(2)}\right)^{-1}\left(\tilde{H}_{u u} \dot{u}+\tilde{H}_{u y} f-\tilde{H}_{y} f_{u}\right)
$$

where $\dot{u}$ is given on the interior of boundary arcs by $\dot{u}=-\left(g_{u}^{(2)}\right)^{-1} g_{y}^{(2)} f$.

The homotopy algorithm is as follows. We denote the current structure of the trajectory by $\mathcal{S}$, i.e. the variable $\mathcal{S}$ indicates the number and order of boundary arcs and touch points. The shooting function associated with the structure $\mathcal{S}$ is denoted by $\mathcal{F}_{\mathcal{S}}$. Moreover, the algorithm uses the two functions below connected with the changes of structure

$$
[\sigma, \omega]=\text { ChangeStruct }(\theta, \mu, \mathcal{S}) \quad \text { and } \quad(\tilde{\theta}, \tilde{\mathcal{S}})=\operatorname{UpdateStruct}(\theta, \mu, \mathcal{S}) .
$$

The function ChangeStruct $(\theta, \mu, \mathcal{S})$ returns $\sigma=$ true if a change in the structure has been detected, i.e. if one of the conditions below is satisfied (the trajectory associated with the structure $\mathcal{S}$, the vector of shooting parameters $\theta$ and the homotopy parameter $\mu$ in the shooting algorithm is denoted by $\left.\left(u_{\mathcal{S}, \theta}^{\mu}, y_{\mathcal{S}, \theta}^{\mu}\right)\right)$ :

$$
\begin{aligned}
\left(g^{\mu}\right)^{(2)}\left(u_{\mathcal{S}, \theta}^{\mu}\left(\tau_{t o}^{i}\right), y_{\mathcal{S}, \theta}^{\mu}\left(\tau_{t o}^{i}\right)\right) & \geq 0 \quad \text { for a touch point } \tau_{t o}^{i}, \\
\max _{t \in[0, T]} g^{\mu}\left(y_{\mathcal{S}, \theta}^{\mu}(t)\right) & >0, \\
\nu_{t o}^{i} & <0 \quad \text { for a touch point } \tau_{t o}^{i}, \\
\tau_{e n}^{i} & >\tau_{e x}^{i} \quad \text { for a boundary } \operatorname{arc}\left(\tau_{e n}^{i}, \tau_{e x}^{i}\right),
\end{aligned}
$$

while $\omega$ is equal to the minimum of $\dot{\eta}=\ddot{\eta}_{2}$ on boundary arcs computed using (24) ( $\omega=$ $+\infty$ if there is no boundary arc). If a change of structure has been detected, i.e. if one of the conditions (25) - (28) holds true, the function UpdateStruct $(\theta, \mu, \mathcal{S})$ returns the new structure $\tilde{\mathcal{S}}$ and a vector of shooting parameters $\tilde{\theta}$ of appropriate dimension with $\tilde{\mathcal{S}}$, obtained as follows: 
- If (25) holds, then $\tilde{\mathcal{S}}$ is obtained from $\mathcal{S}$ by replacing the touch point $\tau_{\text {to }}^{i}$ by a boundary arc, and $\tilde{\theta}$ is obtained from $\theta$ by replacing the touch point $\tau_{t o}^{i}$ and its associated shooting parameters $\nu_{t o}^{i}$ and $\left(y_{t o}^{i}, p_{t o}^{i}\right)$ by a boundary arc, with associated shooting parameters

$$
\begin{gathered}
\tau_{e n}^{i}:=\tau_{t o}^{i}=: \tau_{e x}^{i}, \quad \nu_{e n}^{1, i}:=\nu_{t o}^{i}, \quad \nu_{e n}^{2, i}:=0 \\
\left(y_{e n}^{i}, p_{e n}^{i}\right):=\left(y_{t o}^{i}, p_{t o}^{i}\right)=:\left(y_{e x}^{i}, p_{e x}^{i}\right) .
\end{gathered}
$$

- If (26) holds and (25) does not, set $\tau:=\operatorname{argmax}_{t \in[0, T]} g^{\mu}\left(y_{\mathcal{S}, \theta}^{\mu}(t)\right)$. Then $\tilde{\mathcal{S}}$ is obtained from $\mathcal{S}$ by adding the new touch point $\tau$ and $\tilde{\theta}$ is obtained from $\theta$ by adding the touch point $\tau_{\text {to }}^{i}:=\tau$, a zero jump parameter $\nu_{t_{o}}^{i}:=0$ and the state-costate value at touch point $\left(y_{t o}^{i}, p_{t o}^{i}\right):=\left(y_{\mathcal{S}, \theta}^{\mu}(\tau), p_{\mathcal{S}, \theta}^{\mu}(\tau)\right)$.

- If 27] holds, then $\tilde{\mathcal{S}}$ is obtained from $\mathcal{S}$ by deleting the touch point $\tau_{\text {to }}^{i}$, and $\tilde{\theta}$ is obtained from $\theta$ by deleting the touch point $\tau_{t o}^{i}$, its jump parameter $\nu_{t o}^{i}$ and its associated state-costate value $\left(y_{t o}^{i}, p_{t o}^{i}\right)$.

- If (28) holds, then $\tilde{\mathcal{S}}$ is obtained from $\mathcal{S}$ by replacing the boundary arc $\left(\tau_{e n}^{i}, \tau_{e x}^{i}\right)$ by a touch point, and $\tilde{\theta}$ is obtained from $\theta$ by replacing the shooting parameters associated with the boundary arc $\left(\tau_{e n}^{i}, \tau_{e x}^{i}\right)$ by a touch point and its associated shooting parameters:

$$
\tau_{t o}^{i}:=\tau_{\text {en }}^{i}, \quad \nu_{\text {to }}^{i}:=\nu_{\text {en }}^{1, i}, \quad\left(y_{\text {to }}^{i}, p_{\text {to }}^{i}\right):=\left(y_{\text {en }}^{i}, p_{\text {en }}^{i}\right) .
$$

Finally, the function $\theta=\operatorname{Shooting}\left(\theta_{0}, \mu, \mathcal{F}_{\mathcal{S}}\right)$ returns, if succeeds, a vector $\theta$ solution of $\mathcal{F}_{\mathcal{S}}(\theta, \mu)=0$ obtained e.g. by a Newton algorithm initialized by the value $\theta_{0}$. Combining a classical predictor-corrector algorithm (see [1]) with the changes of structure described above, we obtain Algorithm

The algorithm ends either because $\bar{\mu}=1$ (success), or because the homotopy steplength $\delta$ has been too much reduced (failure), or because $\omega \leq 0$ (the strict complementarity assumption on boundary arcs (23) fails).

The steplength $\delta$ is reduced either when the prediction is judged too bad, or when the correction fails, or at change of structure of the trajectory if the algorithm fails to initialize a vector of shooting parameters for the new structure or does not find the new structure. It is increased after a successfull predictor-corrector step, so that the algorithm adjusts itself the homotopy steplength.

The complementarity condition on boundary arcs fails if a change of structure occurs that was not anticipated by the algorithm. This can happen e.g. if a boundary arc splits into two boundary arcs, or if the function $\Lambda(\tau)$ is positive at a nonreducible touch point $\tau$ (transformation of a touch point into two touch points). Otherwise, it has been shown in [12] that when $\Lambda(\tau)<0$, the uniform strict complementarity holds on emerging boundary arcs.

It was shown in 12 that under some assumptions, this algorithm succeeds in reaching $\mu=1$. It is assumed in particular that only one change in the structure occur at a time.

Remark 6. No constraints on the initial and final state were considered in [12, but the results can be extended if a strong controllability condition is assumed (see [4, section 8]). This condition means that the constraints (2)-(4) are jointly onto.

\section{Model of atmospheric reentry}

\subsection{The optimal control problem}

We use the model of atmospheric reentry of 9], to which we refer for further details. Let us recall that the state is composed of six variables, the radius $r$, velocity $v$, flight path 


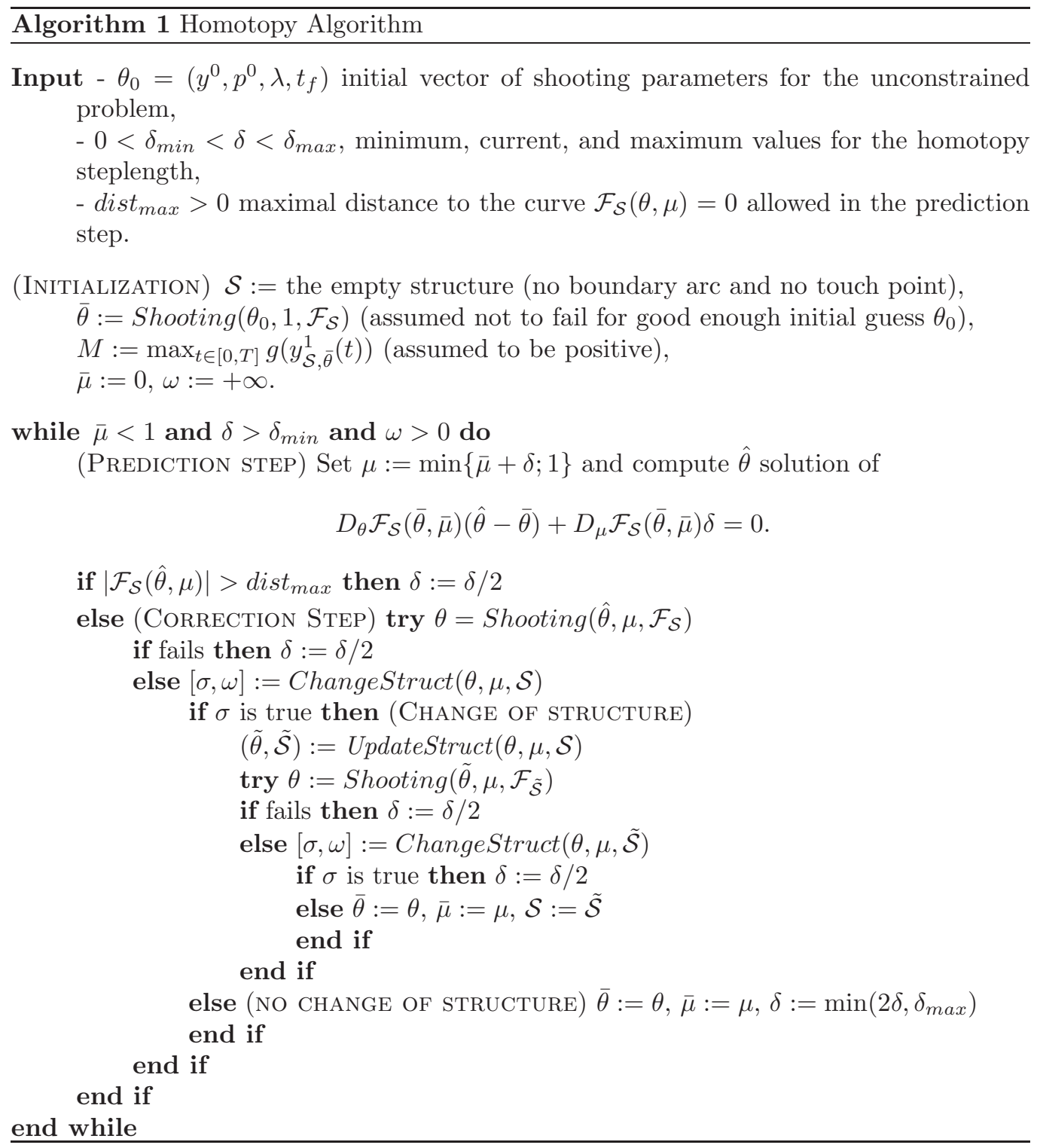


angle $\gamma$, latitude $L$, longitude $l$, and azimuth $\chi$. The control is the bank angle $\beta$ (the angle of attack is fixed following a given incidence profile, see [9 p.135]). The dynamics writes:

$$
\begin{aligned}
\dot{r}= & v \sin \gamma, \\
\dot{v}= & -g \sin \gamma-\frac{S C_{D}}{2 m} \rho v^{2}+\Omega^{2} r \cos L(\sin \gamma \cos L-\cos \gamma \sin L \cos \chi), \\
\dot{\gamma}= & \left(-\frac{g}{v}+\frac{v}{r}\right) \cos \gamma+\frac{S C_{L}}{2 m} \rho v \cos \beta+2 \Omega \cos L \sin \chi \\
& +\Omega^{2} \frac{r}{v} \cos L(\cos \gamma \cos L+\sin \gamma \sin L \cos \chi), \\
\dot{L}= & \frac{v}{r} \cos \gamma \cos \chi \\
i= & \frac{v}{r} \frac{\cos \gamma \sin \chi}{\cos L}, \\
\dot{\chi}= & \frac{S C_{L}}{2 m} \rho \frac{v}{\cos \gamma} \sin \beta+\frac{v}{r} \cos \gamma \tan L \sin \chi+2 \Omega(\sin L-\tan \gamma \cos L \cos \chi) \\
& +\Omega^{2} \frac{r}{v} \frac{\sin L \cos L \sin \chi}{\cos \gamma} .
\end{aligned}
$$

The gravity $g$ and atmospheric density $\rho$ are modeled respectively by

$$
g=\frac{g_{0}}{r^{2}} \quad \text { and } \quad \rho=\rho_{0} e^{\frac{-\left(r-r_{E}\right)}{h_{s}}}
$$

where $r_{E}$ denotes the Earth radius and $g_{0}$ and $h_{s}$ are positive constants whose numerical values can be found - as well as the other constants of the problem - in 9]. The constants $S$ and $m$ denote respectively the reference surface and mass of the shuttle, and $\Omega$ is the Earth rotation velocity. Here the (positive) aerodynamics coefficients $C_{L}$ and $C_{D}$ are interpolated from the tabulated values of 9 as $C^{3}$ functions (piecewise polynomials of order 7) of $r$ and $v$ in order to satisfy assumption (A1).

The objective is to minimize the integral of the thermal flux, as in [7, 9]:

$$
\min \int_{0}^{t_{f}} C_{q} \sqrt{\rho} v^{3} \mathrm{~d} t
$$

where $C_{q}>0$ is a fixed constant. The final time $t_{f}$ is free.

Moreover, we consider the state constraint on the thermal flux, also considered in [3, 17, 9, 13]:

$$
\Phi=C_{q} \sqrt{\rho} v^{3} \leq \Phi_{\max }
$$

with $\Phi_{\max }=7 \cdot 4 \cdot 10^{5} \mathrm{~W} \cdot \mathrm{m}^{-2}$. (A different value $\Phi_{\max }=7 \cdot 173 \cdot 10^{5} \mathrm{~W} \cdot \mathrm{m}^{-2}$ was considered in [9. We explain later in Rem. 11] why our homotopy algorithm does not allow us to solve the problem for $\Phi_{\max }<7.4 .10^{5}$.)

Finally, the problem is subject to the boundary conditions given in Table 1 (9]).

\begin{tabular}{|l|c|c|}
\hline & Initial conditions & Final conditions \\
\hline Altitude $h=r-r_{E}$ & $119.82 \mathrm{~km}$ & $15 \mathrm{~km}$ \\
Velocity $v$ & $7404.95 \mathrm{~m}^{-1}$ & $445 \mathrm{~m} . \mathrm{s}^{-1}$ \\
Flight angle $\gamma$ & $-1.84^{\circ}$ & free \\
Latitude $L$ & 0 & $10.99^{\circ}$ \\
Longitude $l$ & free & $166.48^{\circ}$ \\
Azimuth $\chi$ & free & free. \\
\hline
\end{tabular}

Table 1: Boundary conditions 
Remark 7. It can be seen that the right-hand side of the dynamics (29)-34) does not depends on the longitude $l$, which does not appear in the cost (35) nor in the constraint (36). Since the initial longitude is free, the variable $l$ can be omitted and computed afterwards once the optimal trajectory has been obtained by integrating backwards (33) from its final value $l_{t_{f}}=166.48$ prescribed in Table \(see 9]).

In what follows, we denote by $y=(r, v, \gamma, L, \chi)$ the state variable and by $u$ or $\beta$ the scalar control.

\subsection{Assumptions}

In order to apply the homotopy algorithm of [12, it is required that assumptions (A1)(A3) hold. Assumption (A1) is satisfied by our model, and we will discuss in this section the validity of assumptions (A2) and (A3).

Regularity of the Hamiltonian Let $(u, y)$ denote an optimal trajectory. We have that

$$
\mathcal{H}_{u}=-p_{\gamma} \frac{S C_{L}}{2 m} \rho v \sin \beta+p_{\chi} \frac{S C_{L}}{2 m} \rho \frac{v}{\cos \gamma} \cos \beta
$$

where $\mathcal{H}$ denotes the generalized Hamiltonian (7). Let us assume that (this will be checked in the numerical simulation)

Assumption (H0) In the flight domain, $\cos \gamma>0$.

If $p_{\gamma}$ and $p_{\chi}$ do not vanish together, the (unique modulo $2 \pi$ ) control $\beta$ minimizing the Hamiltonian over $U=\mathbb{R}$, i.e. satisfying (10), is given by

$$
\cos \beta=-\frac{\cos \gamma p_{\gamma}}{\sqrt{\cos \gamma^{2} p_{\gamma}^{2}+p_{\chi}^{2}}}, \quad \sin \beta=-\frac{p_{\chi}}{\sqrt{\cos \gamma^{2} p_{\gamma}^{2}+p_{\chi}^{2}}} .
$$

(The control $\beta$ is not uniquely determined, but since it is involved in the equations of the problem only by its sine and cosine, it is sufficient that the two above quantities are uniquely defined). However, because of our final boundary conditions and the transversality condition (12), we have that $p_{\gamma}\left(t_{f}\right)=p_{\chi}\left(t_{f}\right)=0$. We shall make the assumption below:

Assumption (H1) For all $t \in\left[0, t_{f}\right)$, either $p_{\gamma}(t) \neq 0$ or $p_{\chi}(t) \neq 0$.

This assumption will be checked numerically since, as we will see, we have $p_{\chi}(t)<0$ on $\left(0, t_{f}\right)$ and $p_{\gamma}(0) \neq 0$ (see Fig. 2(b) to $5(\mathrm{~b})$.

Lemma 8. Let $(u, y)$ be a Pontryagin extremal. If assumption (HO)-(H1) hold, then $u$ is continuous over $\left[0, t_{f}\right)$.

Proof. On $\left[0, t_{f}\right)$, the control $u$ that minimizes the Hamiltonian is given by (37). Since the state constraint (36) does not depend on $\gamma$ nor on $\chi$, the associated costate components, respectively $p_{\gamma}$ and $p_{\chi}$, are continuous over $\left[0, t_{f}\right]$ by (9). The result follows.

Under assumptions (H0)-(H1), the control $\beta$ can be computed as a smooth function of the state and costate by (37) on $\left[0, t_{f}\right)$. Moreover, at the minimizing control (37), we have that

$$
\mathcal{H}_{u u}=H_{u u}=\frac{S C_{L}}{2 m} \rho \frac{v}{\cos \gamma} \sqrt{\cos \gamma^{2} p_{\gamma}^{2}+p_{\chi}^{2}} .
$$

Therefore, the strenghtened Legendre-Clebsch condition (14) holds on $\left[0, t_{f}-\varepsilon\right]$ for every $\varepsilon>0$, i.e. for every $\varepsilon>0$ there exists $\alpha>0$ such that $H_{u u} \geq \alpha$ on $\left[0, t_{f}-\varepsilon\right]$. Since the state constraint is not active at final time $t_{f}$, the homotopy algorithm 1 is valid to detect the structure of the trajectory as long as assumption (H1) remains satisfied (see Remark [1]. 
Regularity of the state constraint The constraint on the thermal flux (36) is a second-order state constraint. The second-order time derivative of the state constraint is given by

$$
g^{(2)}=A+B \cos \beta+C \sin \beta
$$

where $A=A(y), B=B(y)$, and $C=C(y)$ are functions of the state and can be computed either by hand or using formal calculus (see [9. p.122]). If $0<B^{2}+C^{2}$ and $A^{2} \leq B^{2}+C^{2}$, the control on boundary arcs can be computed by

$\cos \beta=\frac{-A B+\sigma C \sqrt{B^{2}+C^{2}-A^{2}}}{B^{2}+C^{2}}, \quad \sin \beta=\frac{-A C-\sigma B \sqrt{B^{2}+C^{2}-A^{2}}}{B^{2}+C^{2}}, \quad \sigma \in\{-1,1\}$.

The solution with $\sigma=1$ is convenient in order to have a continuous control, which is a necessary optimality condition by Lemma 8 Moreover, we have that

$$
g_{u}^{(2)}=\sigma \sqrt{B^{2}+C^{2}-A^{2}} .
$$

We shall therefore make the assumption below, which will be checked in the numerical simulations:

Assumption (H2) In the neighborhood of the contact set of the state constraint, we have $A^{2}<B^{2}+C^{2}$.

This assumption implies that (A3) holds.

Finally, we will assume that the extremals of the problem are normal $\left(p_{0}=1\right)$.

\section{Resolution of the unconstrained problem}

In order to apply the homotopy algorithm, it is required that a sufficiently good initial guess for the initial costate of the unconstrained problem can be provided in order to make the (simple) shooting algorithm converge. We detail in this section how we proceed to initialize the unconstrained problem.

\subsection{Resolution of the reduced problem in dimension 3}

If we neglect the Earth rotation, i.e. $\Omega=0$, then the problem reduces to a problem with only three state variables $\hat{y}=(r, v, \gamma)$ and control $\hat{u}=\cos \beta \in U=[-1,1]$ (see [10, 8]):

Minimize $\int_{0}^{t_{f}} C_{q} \sqrt{\rho} v^{3} \mathrm{~d} t$ subject to the state equation

$$
\begin{aligned}
\dot{r} & =v \sin \gamma \\
\dot{v} & =-g \sin \gamma-\frac{S C_{D}}{2 m} \rho v^{2}, \\
\dot{\gamma} & =\left(-\frac{g}{v}+\frac{v}{r}\right) \cos \gamma+\frac{S C_{L}}{2 m} \rho v \hat{u} .
\end{aligned}
$$

Moreover, the problem is subject to the boundary conditions for $r, v$ and $\gamma$ given in Table (1)

It was shown in [10, 8, by a geometric analysis that the optimal trajectory for the above reduced problem in dimension 3 is composed by an arc with $\hat{u}=-1$ followed by an arc with $\hat{u}=+1$.

In order to obtain the solution of the reduced problem, we proceed as in [9] and introduce the switching time $t_{\text {switch }}$ and the free final time $t_{f}$. Given a couple $\left(t_{\text {switch }}, t_{f}\right)$, starting from the initial conditions $\hat{y}^{0}=\left(r_{0}, v_{0}, \gamma_{0}\right)$ of Table 1 we integrate (39)- (41) with the control $\hat{u}=-1$ on $\left(0, t_{\text {switch }}\right)$ and $\hat{u}=+1$ on $\left(t_{\text {switch }}, t_{f}\right)$ and search for a solution $\left(t_{\text {switch }}^{*}, t_{f}^{*}\right)$ that satisfies the final conditions $r\left(t_{f}\right)=r_{t_{f}}$ and $v\left(t_{f}\right)=v_{t_{f}}$ of Table 1 In 
this way the optimal trajectory solution of the reduced problem in dimension 3 can be obtained without introducing the adjoint variables.

In order to determine the latter, we introduce the unknown initial costate $\hat{p}^{0}=$ $\left(p_{r}^{0}, p_{v}^{0}, p_{\gamma}^{0}\right)=\left(p_{r}(0), p_{v}(0), p_{\gamma}(0)\right)$. Since the Hamiltonian of the reduced problem $\hat{H}$ is given by

$$
\hat{H}=p_{\gamma} \frac{S C_{L}}{2 m} \rho v \hat{u}+\text { a function of }(\hat{y}, \hat{p}),
$$

Pontryagin's minimum principle (10) yields that

$$
\hat{u}(t)= \begin{cases}-1 & \text { if } p_{\gamma}(t)>0 \\ +1 & \text { if } p_{\gamma}(t)<0\end{cases}
$$

Since $p_{\gamma}$ is continuous, (42) together with (12) and Rem. 3implies that the three following necessary conditions have to be satisfied:

$$
p_{\gamma}\left(t_{\text {switch }}^{*}\right)=0, \quad p_{\gamma}\left(t_{f}^{*}\right)=0, \quad H(\hat{u}(0), \hat{y}(0), \hat{p}(0))=0 .
$$

Since the initial state $\hat{y}^{0}$ is known, as well as $\hat{u}(0)=-1$, the last condition allows us to express $p_{\gamma}^{0}$ in function of $p_{r}^{0}$ and $p_{v}^{0}$ by

$$
p_{\gamma}^{0}=-\left(\Phi\left(\hat{y}^{0}\right)+p_{r}^{0} f_{r}\left(\hat{y}^{0}\right)+p_{v}^{0} f_{v}\left(\hat{y}^{0}\right)\right) / f_{\gamma}\left(\hat{u}(0), \hat{y}^{0}\right) .
$$

By a shooting algorithm (initialized by zero), integrating the costate equations we easily obtain initial values $p_{r}^{0}$ and $p_{v}^{0}$ in order to satisfy the two first conditions of [43).

Remark 9. In order to ensure that the obtained solution is a Pontryagin extremal, we have to check that (42) is satisfied, i.e. $p_{\gamma} \geq 0$ on the first arc where $\hat{u}=-1$ and $p_{\gamma} \leq 0$ on the second arc where $\hat{u}=+1$. There are indeed several couples $\left(t_{\text {switch }}, t_{f}\right)$ that enable to reach the desired final conditions $\left(r_{t_{f}}, v_{t_{f}}\right)$ but that are not optimal since we do not have $p_{\gamma} \leq 0$ on the arc where $\hat{u}=+1$, as it is shown in Table 2] Here only the last solution (solution 6$)$ satisfies the conditions $p_{\gamma} \geq 0$ on $\left(0, t_{\text {switch }}\right)$ and $p_{\gamma} \leq 0$ on $\left(t_{\text {switch }}, t_{f}\right)$.

\begin{tabular}{|l|c|c|c|}
\hline & $t_{\text {switch }} \approx$ & $t_{f} \approx$ & $\int_{0}^{t_{f}} C_{q \sqrt{\rho}} v^{3} \mathrm{~d} t \approx$ \\
\hline Solution 1 & 166.16 & 1324.95 & $3.718 .10^{8}$ \\
Solution 2 & 183.22 & 1219.52 & $3.080 .10^{8}$ \\
Solution 3 & 201.10 & 899.50 & $1.837 .10^{8}$ \\
Solution 4 & 210.82 & 451.12 & $1.303 .10^{8}$ \\
Solution 5 & 213.42 & 333.73 & $1.258 .10^{8}$ \\
Solution 6 & 214.06 & 242.61 & $1.253 .10^{8}$ \\
\hline
\end{tabular}

Table 2: Couples $\left(t_{\text {switch }}, t_{f}\right)$ that enable to reach the final condition $\left(r_{t_{f}}, v_{t_{f}}\right)$ with the control $\hat{u}=-1$ on $\left(0, t_{\text {switch }}\right)$ and $\hat{u}=+1$ on $\left(t_{\text {switch }}, t_{f}\right)$ in the reduced problem and value of the associated cost function.

\subsection{Resolution of the unconstrained problem}

Now we go back to the problem in dimension 5 and first consider the case when $\Omega=0$. Since the trajectory $(r, v, \gamma)$ and control $\beta$ solution of the reduced problem in dimension 3 are known, it is then easy to introduce the unknown initial azimuth $\chi^{0}=\chi(0)$ and integrate the equations (32) and (34) with $\Omega=0$ so as to satisfy the remaining final condition $L\left(t_{f}\right)=L_{t_{f}}$. Since $\left(p_{r}^{0}, p_{v}^{0}, p_{\gamma}^{0}\right)$ are known and $p_{L}=p_{\chi} \equiv 0$, we have a solution of the unconstrained problem when $\Omega=0$.

When the Earth rotation velocity $\Omega$ is not neglected, we first compute like in $[9$ an approximate solution by assuming that the control is such that $\cos \beta=-1$ on $\left(0, t_{\text {switch }}\right)$ 
and $\cos \beta=+1$ on $\left(t_{\text {switch }}, t_{f}\right)$. Here $t_{\text {switch }}$ is a free parameter, as well as the final time $t_{f}$. Using the values of parameters solutions of the problem with $\Omega=0$ as initial guess, we obtain a zero of the shooting function

$$
\left(\begin{array}{c}
\chi^{0} \\
p_{r}^{0} \\
p_{v}^{0} \\
p_{\gamma}^{0} \\
p_{L}^{0} \\
t_{s w i t c h} \\
t_{f}
\end{array}\right) \mapsto\left(\begin{array}{c}
H(u(0), y(0), p(0)) \\
r\left(t_{f}\right)-r_{t_{f}} \\
v\left(t_{f}\right)-v_{t_{f}} \\
L\left(t_{f}\right)-L_{t_{f}} \\
p_{\gamma}\left(t_{s w i t c h}\right) \\
p_{\gamma}\left(t_{f}\right) \\
p_{\chi}\left(t_{f}\right)
\end{array}\right)
$$

where the state and costate equations are integrated with the control $\cos \beta=-1$ on $\left(0, t_{\text {switch }}\right)$ and $\cos \beta=+1$ on $\left(t_{\text {switch }}, t_{f}\right)$. In this way we obtain a trajectory and costate satisfying the state and costate equations (8)-(9) and transversality condition (12) as well as (13). Only the minimum principle (10) is not satisfied.

Now we arrive to the last step of our initialization procedure. We consider the simple shooting function

$$
\left(\begin{array}{c}
\chi^{0} \\
p_{r}^{0} \\
p_{v}^{0} \\
p_{\gamma}^{0} \\
p_{L}^{0} \\
t_{f}
\end{array}\right) \mapsto\left(\begin{array}{c}
H(u(0), y(0), p(0)) \\
r\left(t_{f}\right)-r_{t_{f}} \\
v\left(t_{f}\right)-v_{t_{f}} \\
L\left(t_{f}\right)-L_{t_{f}} \\
p_{\gamma}\left(t_{f}\right) \\
p_{\chi}\left(t_{f}\right)
\end{array}\right)
$$

where the state and costate equations are integrated with the control $\beta$ given as a function of state and costate by (37). The value $\left(\chi^{0}, p_{r}^{0}, p_{v}^{0}, p_{\gamma}^{0}, p_{L}^{0}, t_{f}\right)$ previously obtained as a zero of (44) provides a sufficiently good initial guess to make the simple shooting algorithm converge, and we thus obtain a solution of the unconstrained problem, starting point of the homotopy algorithm. The solution and multipliers of the unconstrained problem are plotted in Fig. 2

\section{Resolution of the problem with state constraint}

Now we have a solution of the problem without the state constraint, we apply the homotopy algorithm 1 to our problem with the constraint (36) on the thermal flux. A sequence of problems $\left(\mathcal{P}^{\mu}\right)$ for $\mu \in[0,1]$ is solved, with state constraint

$$
\Phi=C_{q} \sqrt{\rho} v^{3} \leq \Phi_{\mu}:=\mu \Phi_{\max }+(1-\mu) \Phi_{\text {uncons }}
$$

where $\Phi_{\text {uncons }}$ denotes the maximum of the thermal flux for the optimal solution of the unconstrained problem. We detail below the results of the algorithm.

\section{$5.1 \quad$ Results}

When the value of the homotopy parameter $\mu$ is increased from 0 to 1 , the algorithm detects three changes in the structure of the constraint detailed in Table 3

The thermal flux is plotted in Fig. [ 1 for the unconstrained problem, the final solution and at two intermediate solutions when changes in the structure are detected: The first one has a nonessential touch point and an essential one and the second has a nonreducible touch point and a reducible one.

The state, control and multipliers associated with those four solutions are plotted in Fig. 2 to 5 


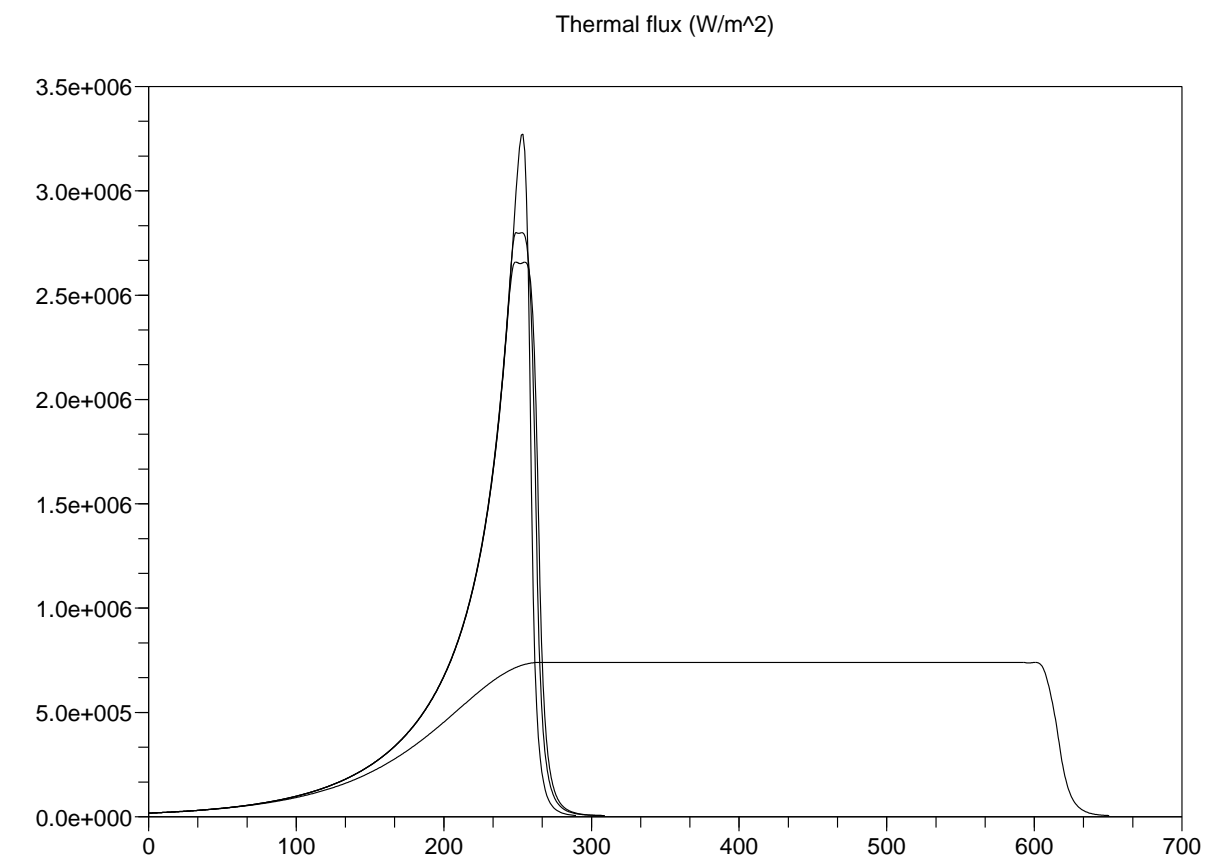

(a) Thermal flux

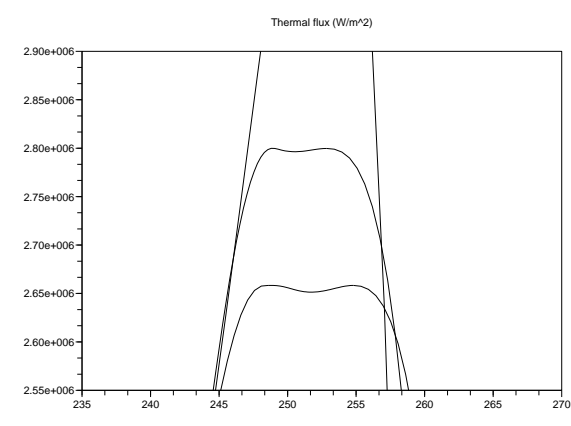

(b) Zoom on the two intermediate solutions

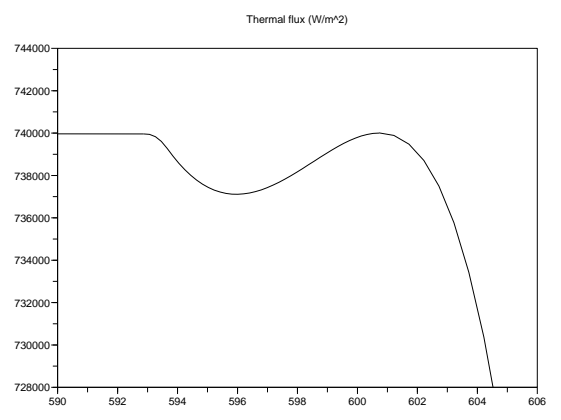

(c) Zoom on the final solution

Figure 1: Thermal flux at the different changes of structure and at the final solution, abscissa is time ( $\mathrm{s})$. 

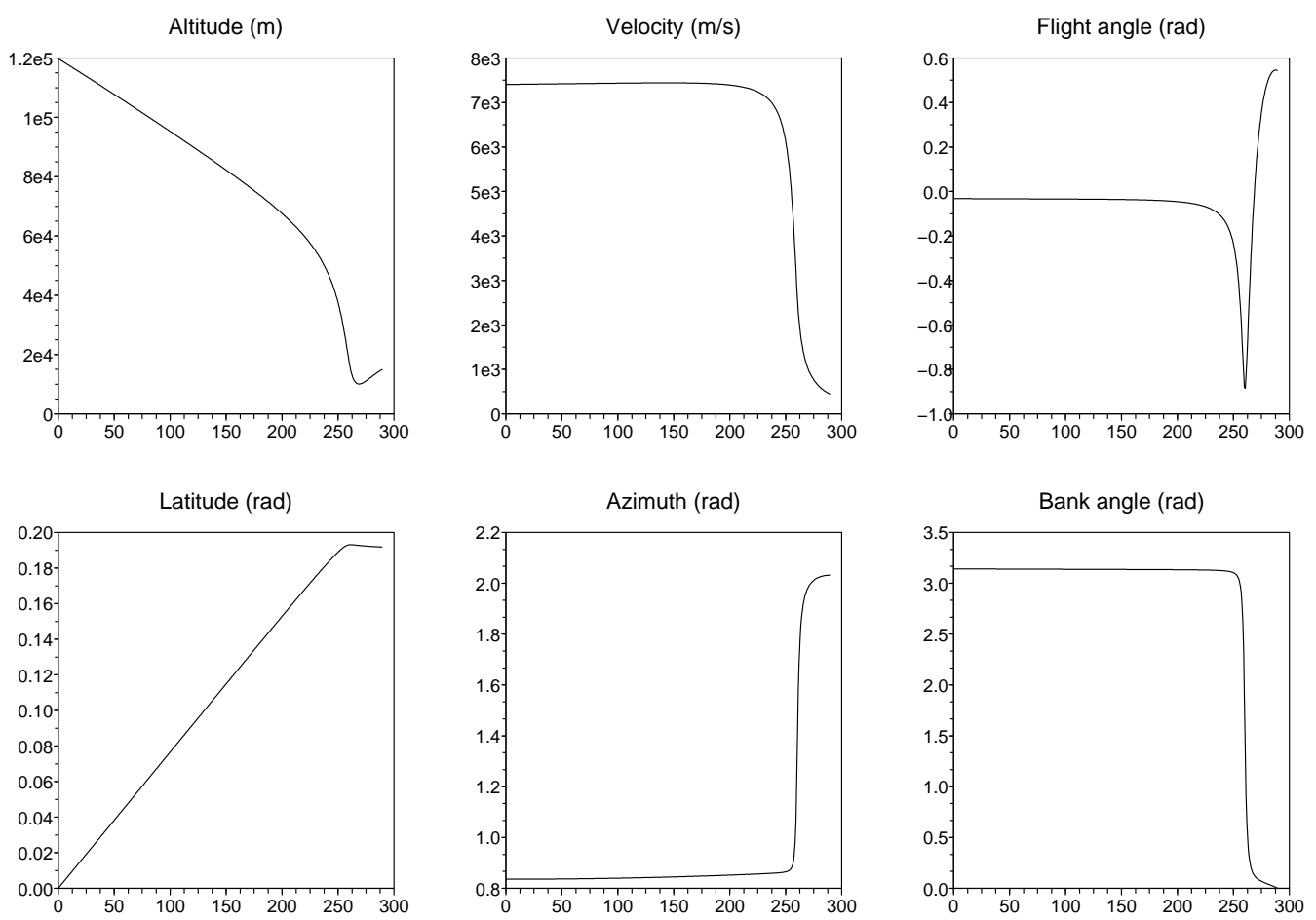

(a) State and control
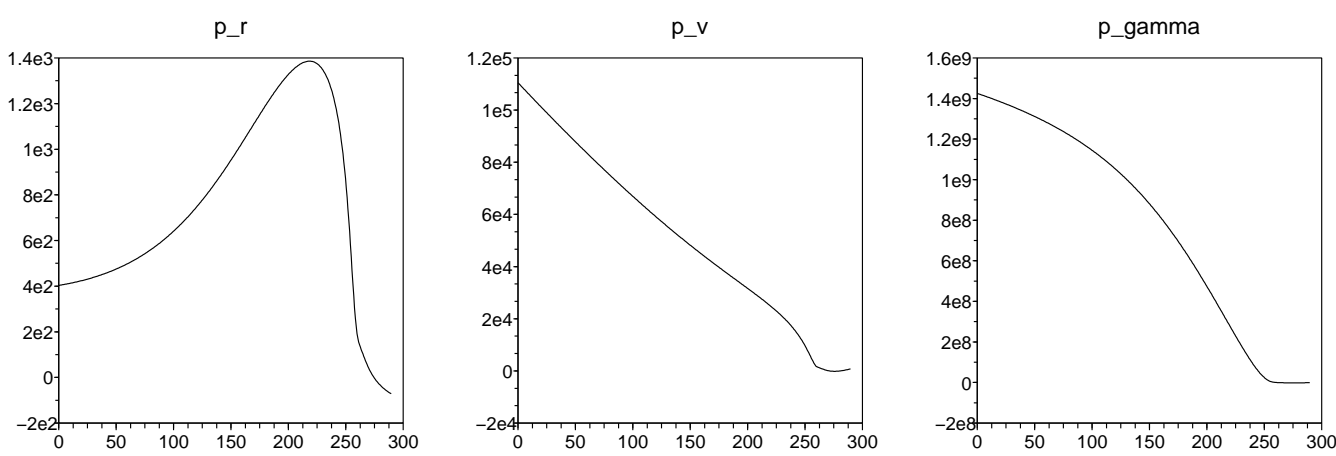

P_L

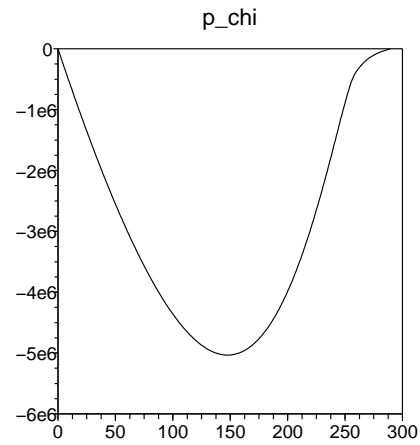

(b) Costate

Figure 2: Solution and multipliers of the unconstrained problem, abscissa is time (s). 

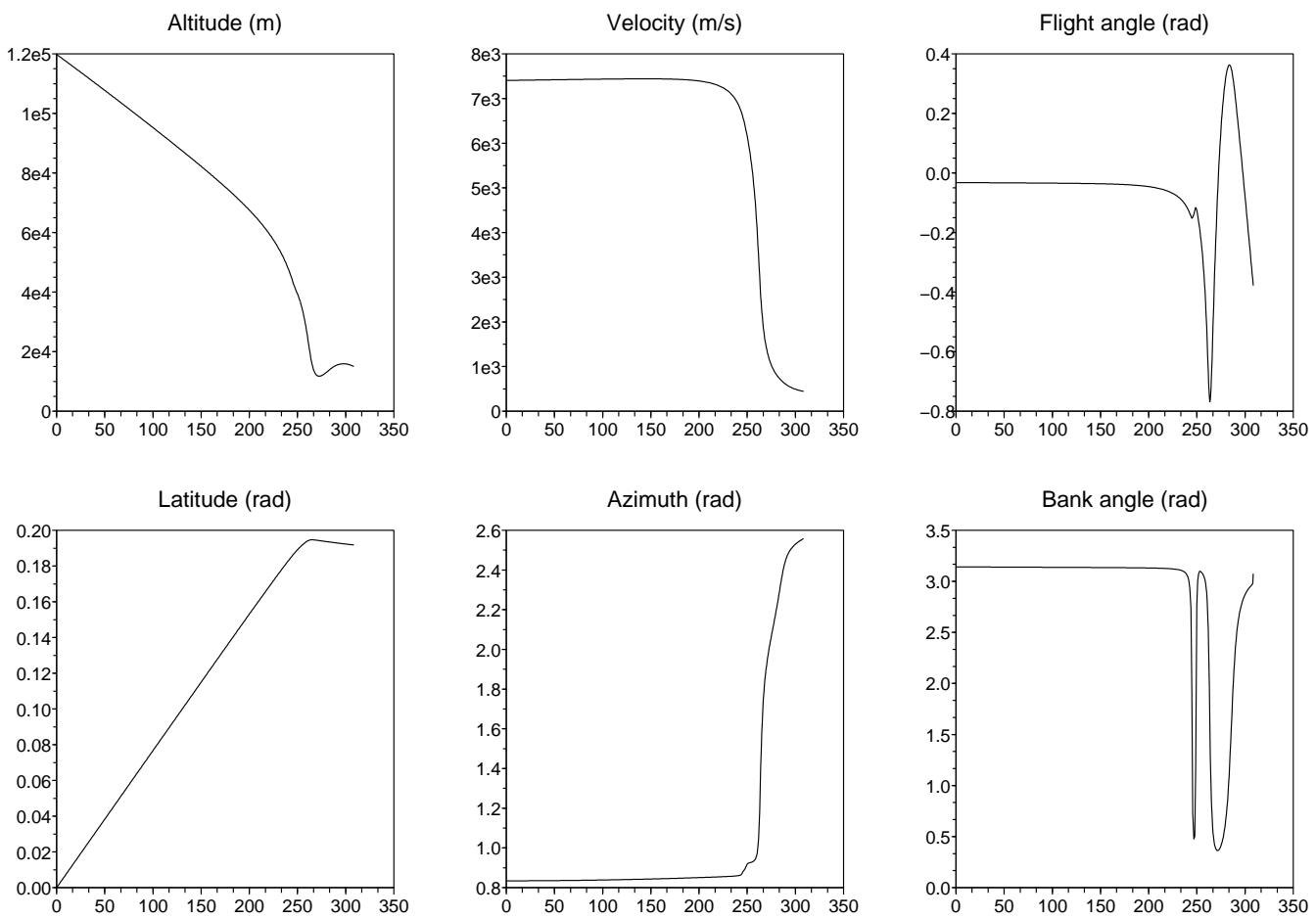

(a) State and control
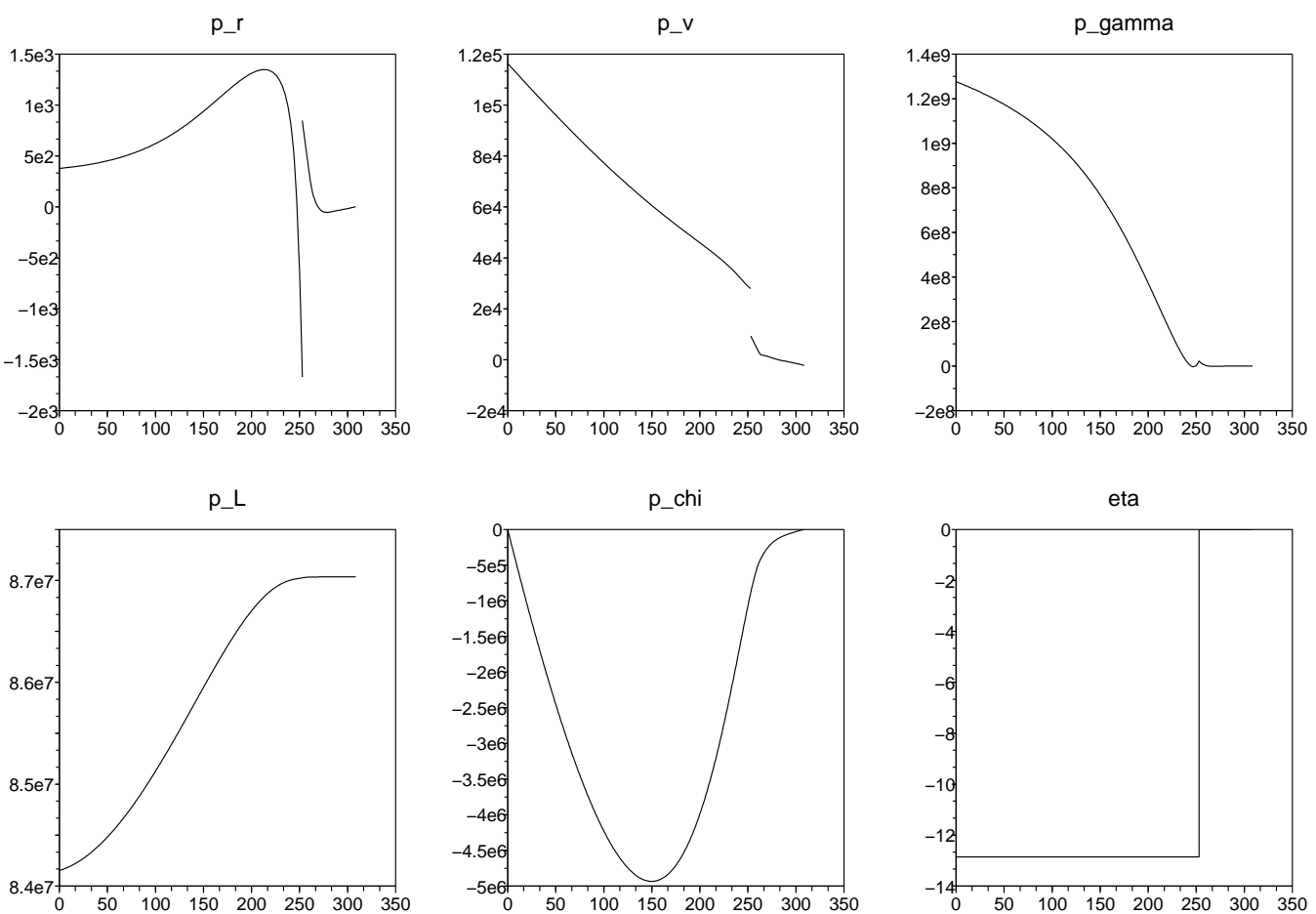

(b) Costate $p$ and state constraint multiplier $\eta$

Figure 3: Solution and multipliers of the constrained problem with $\Phi_{\mu} \approx 2.65 .10^{6} \mathrm{~W} \cdot \mathrm{m}^{-2}$ (two touch points, the first one being nonessential), abscissa is time (s).

$\mathrm{RR} \mathrm{n}^{\circ} 6627$ 

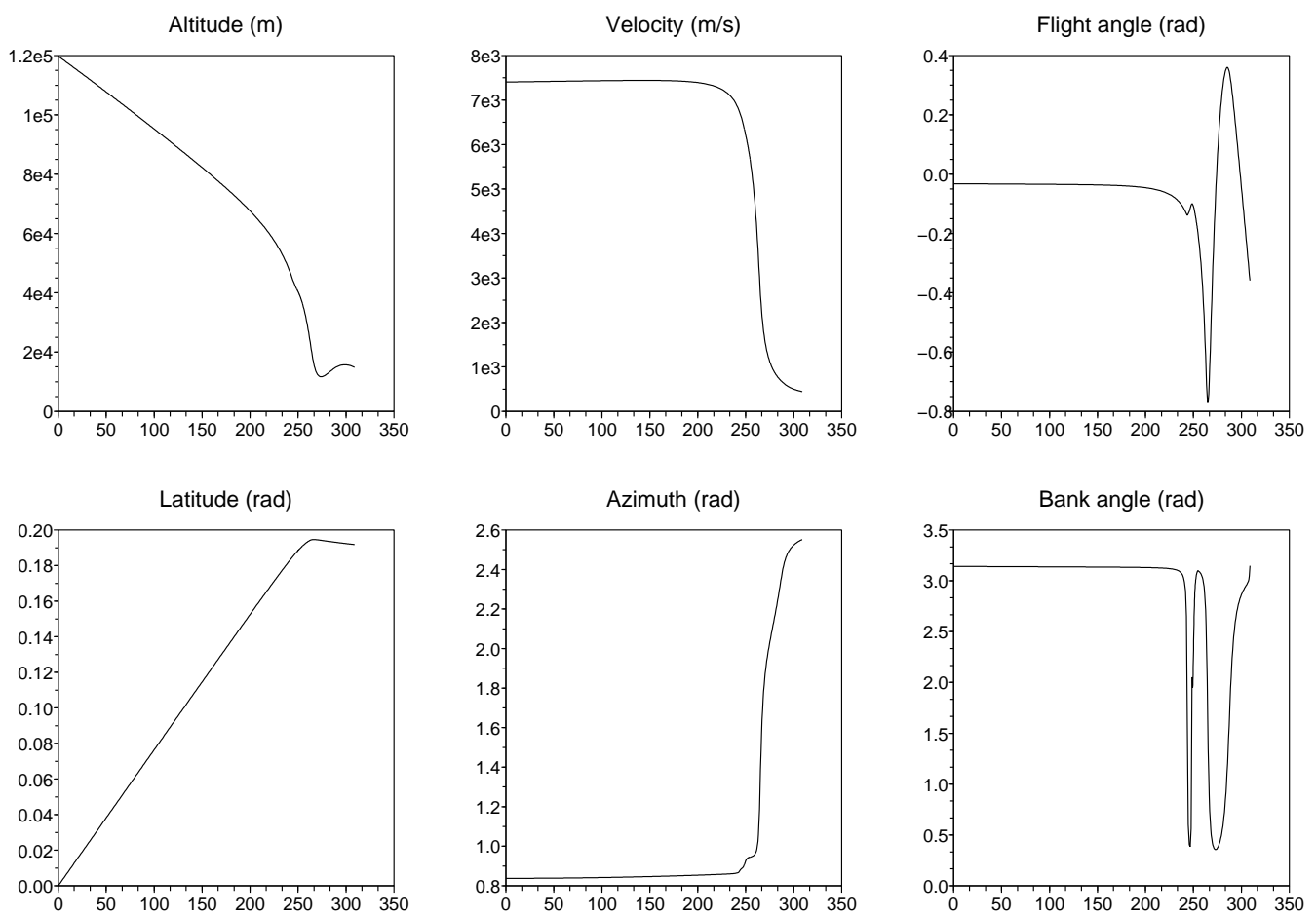

(a) State and control
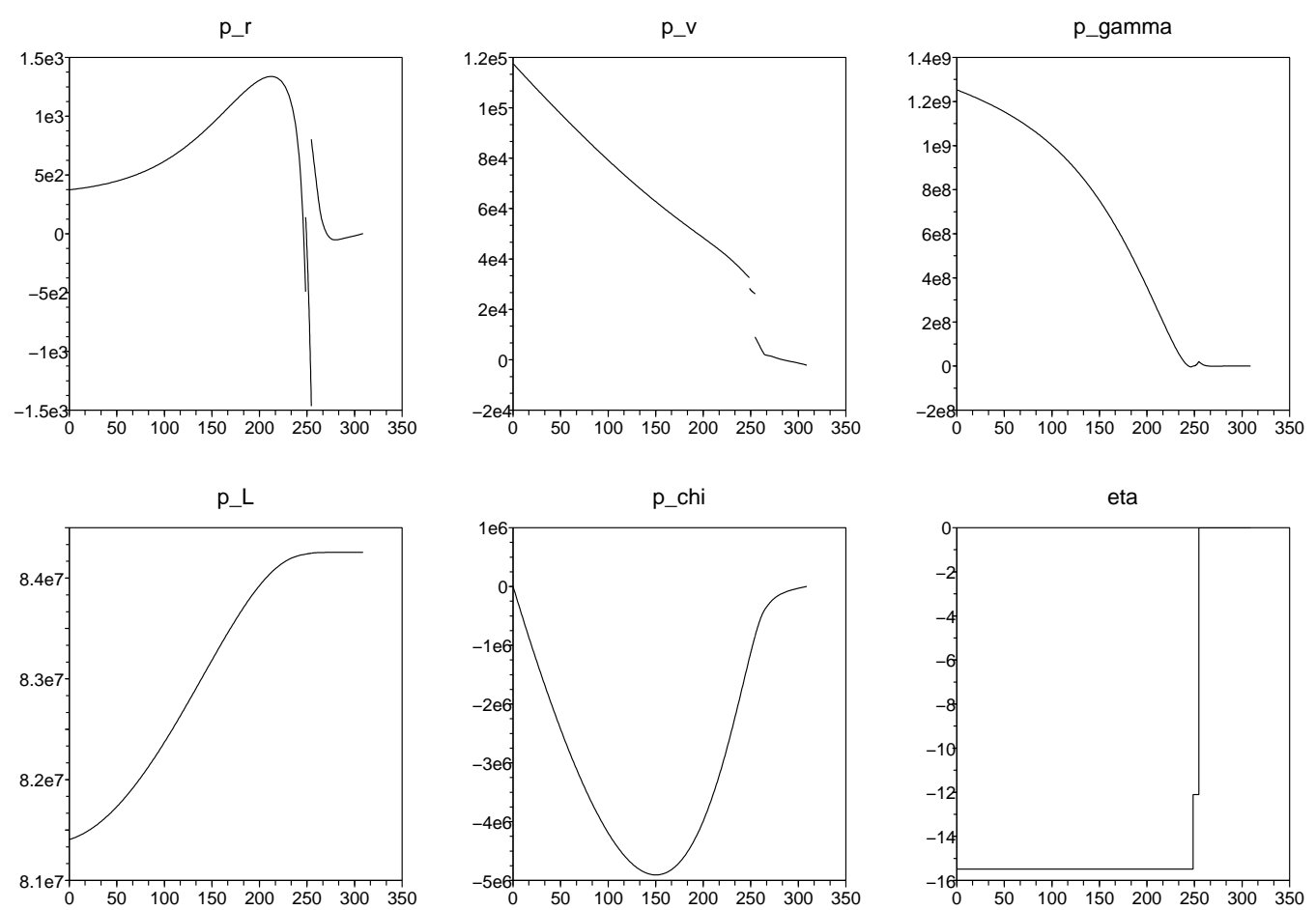

(b) Costate $p$ and state constraint multiplier $\eta$

Figure 4: Solution and multipliers of the constrained problem with $\Phi_{\mu} \approx 2.79 .10^{6}$ (two touch points, the first one being nonreducible), abscissa is time (s). 

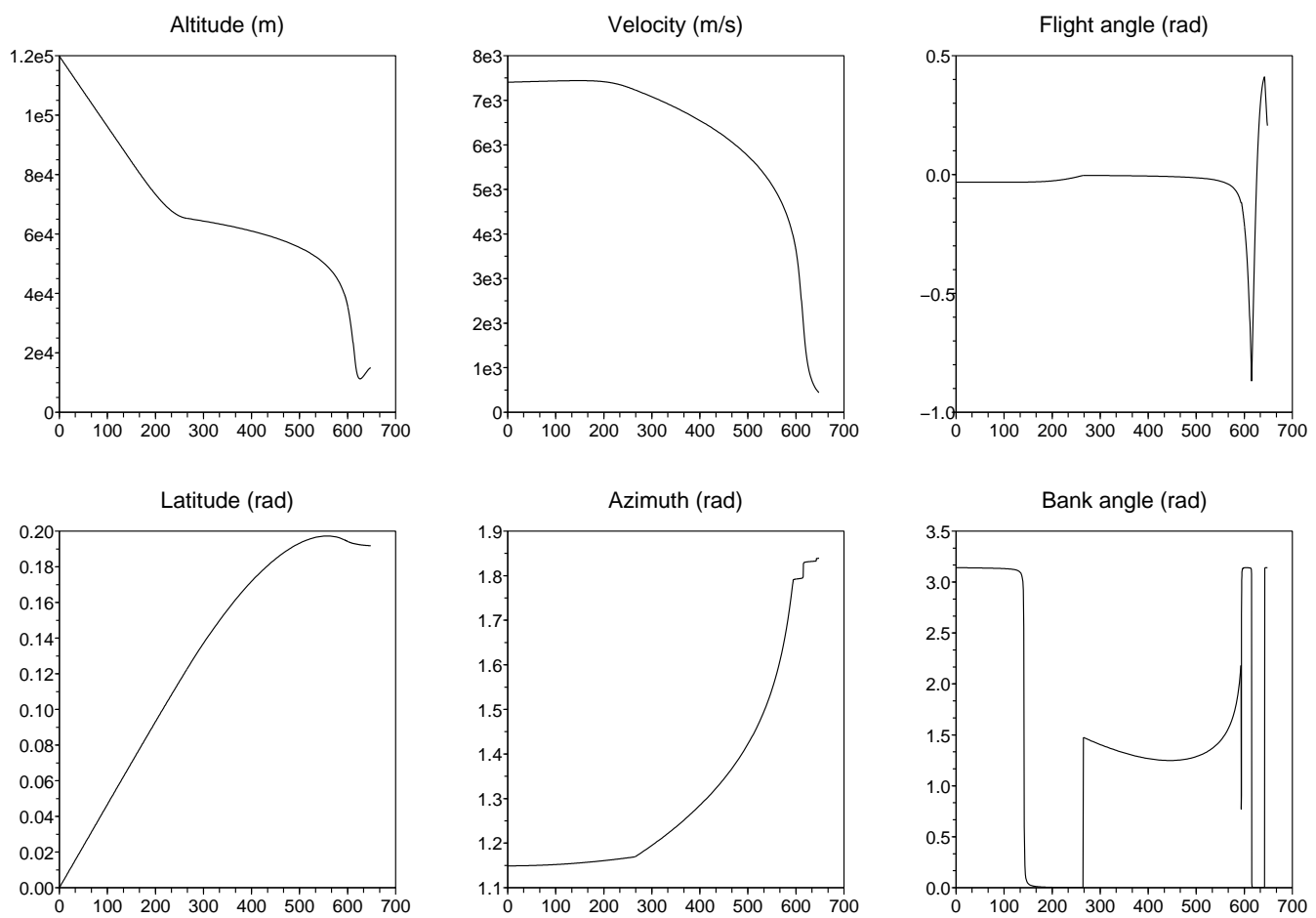

(a) State and control
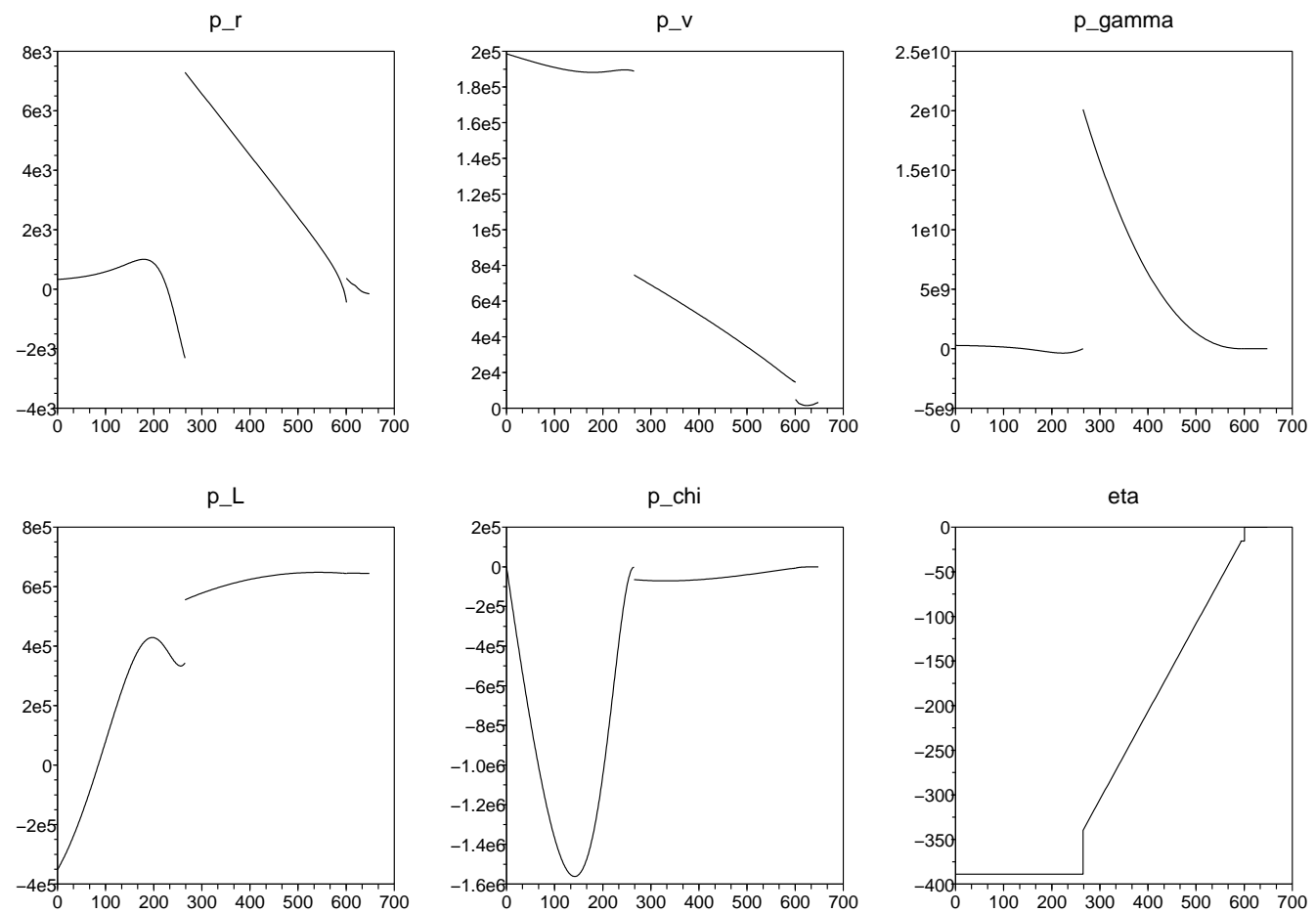

(b) Alternative costate $p_{2}$ and state constraint multiplier $\eta$

Figure 5: Solution and multipliers of the constrained problem with $\Phi_{\mu}=7.4 .10^{5}$ (one boundary arc and one touch point), abscissa is time (s).

$\mathrm{RR} \mathrm{n}^{\circ} 6627$ 


\begin{tabular}{|c|c|c|c|}
\hline$\Phi_{\mu} \approx$ & Event detected & at $\tau \approx$ & New structure of the trajectory \\
\hline $3.27 .10^{6}$ & nonessential touch point & $253 \mathrm{~s}$ & one touch point \\
\hline $2.79 .10^{6}$ & nonessential touch point & $249 \mathrm{~s}$ & two touch points \\
\hline $2.65 .10^{6}$ & nonreducible touch point & $248 \mathrm{~s}$ & $\begin{array}{c}\text { one boundary arc } \\
\text { and one touch point }\end{array}$ \\
\hline
\end{tabular}

Table 3: Changes in the structure of the trajectory.

Remark 10. We can check on Fig. 5(b) (as well as all along the homotopy path) that $\eta$ is strictly increasing on the boundary arc, and therefore the strict complementarity assumption on boundary arc (23) is satisfied.

Remark 11. If we try to pursue the homotopy beyond the value $\Phi_{\max }=7.4 .10^{5}$, we find that $p_{\gamma}\left(\tau_{e n}\right)=p_{\chi}\left(\tau_{e n}\right)=0$ i.e. assumption (H1) is no longer satisfied at entry time of boundary arc. In that case the continuity of the control at entry time is not ensured and the homotopy algorithm [1 is no longer appropriate to solve the problem. It is not even known what an optimality condition for the entry time is when the control enters nonlinearly and the strenghtened Legendre-Clebsch condition does not hold.

Remark 12. In [9], an additional constraint on the normal acceleration was considered. We check here that our final solution of Fig. 5 violates indeed this constraint near the end of the trajectory. This may explain why our solution differs from the one obtained in [9].

\subsection{Comments on numerical implementation}

Convergence results The computation was done using Scilab 4.1.2 1]. A total of 198 iterations (prediction-correction step) are needed due to the high sensitivity of the problem: 26 in the first phase (one touch point), 5 in the second phase (two touch points), and 167 in the last phase (one boundary arc and one touch point). This leads to a rather long total computational time (several hours). Many iterations occur at the very end of the homotopy path since the problem becomes close to singular (see Rem. 111). In order to reach the value $\Phi_{\max }=7 \cdot 5 \cdot 10^{5}$, only a total of 130 iterations is needed.

The procedure to adapt the length of the homotopy stepsize in Algorithm 1 proved to be rather efficient, 74 iterations needed a reduction of the steplength, specially near the end of the homotopy path.

The changes of structure in the trajectory and initialization of the shooting parameters associated with touch points and boundary arc are handled automatically by the algorithm as explained in section 2.2 and pose no additional difficulty in term of convergence. Below we give some details on the implementation

The shooting algorithm We consider a slightly modified shooting function to that of section 2.2 by considering as unknown shooting parameters the duration of each arc rather than the junction and final times themselves. The duration of each arc is then normalized to 1 by a change of time. In this way we gain numerical stability and in addition, the Jacobian of the shooting function is easier to compute (see the next paragraph). In the shooting algorithm, we use a classical Newton algorithm with Armijo line search. If the homotopy steplength $\delta$ is small enough, the line search is not active.

Computation of the Jacobian The Jacobian of the shooting function is computed by integration of the linearized system (see e.g. [6, Lemma 3.7]). This requires the precomputation of the derivatives of the functions of the problem until order 2 (until order 3

\footnotetext{
${ }^{1}$ http://www.scilab.org/
} 
for the state constraint), which has been done here by hands but can also be done using formal calculus or automatic differentiation. This effort is rewarded by a better accuracy, improving the convergence of the shooting algorithm, and a gain in the computational time, both in the prediction and correction step, with comparison to a Jacobian computed by finite differences (centered scheme). An accurate computation of the Jacobian is essential to successfully follow the homotopy path.

Integrator An integrator with fixed step, Runge-Kutta of order 4, is used. This gives a better convergence of the shooting algorithm than an integrator with a variable step. This seems to be due to the fact that the Jacobian of the shooting function can be computed more accurately when the integration step is fixed.

Scaling and preconditioning It is known that in order to limit numerical errors, all the state and costate variables have to be scaled in order to vary e.g. in the interval $[-1,1]$. The jump parameters and duration of each arc have to be scaled as well. This can be done by an efficient preconditioning of the Jacobian of the shooting function. More precisely, in order to compute Newton's direction in the shooting algorithm, instead of solving $A d=b$ where $A:=D_{\theta} \mathcal{F}_{\mathcal{S}}(\theta, \mu)$ and $b:=-\mathcal{F}_{\mathcal{S}}(\theta, \mu)$, we set $B:=A D$ where $D$ is a (given) diagonal matrix with $i$-th element $d_{i i}=10^{k}$ if the $i$-th component of $\theta$ is of order $10^{k}$, solve $B \hat{d}=b$ and then $d:=D \hat{d}$. This simple manipulation considerably improve the convergence of the shooting algorithm. Scaling and preconditioning have to be updated in the course of the algorithm when the homotopy steplength becomes too small, specially in the last phase of the algorithm when the structure is composed of a boundary arc and a touch point. Finally, the use of $Q R$-decompositions to solve the linear systems in the prediction and correction steps (see [1, Chapter 4]) is also an important factor to improve numerical accuracy.

Acknoledgments The author is grateful to Pierre Martinon for helpful discussions about numerical homotopy methods and to Emmanuel Trélat for his remarks on the paper.

\section{References}

[1] E. L. Allgower and K. Georg. Introduction to numerical continuation methods, volume 45 of Classics in Applied Mathematics. Society for Industrial and Applied Mathematics (SIAM), Philadelphia, PA, 2003. Reprint of the 1990 edition [Springer-Verlag, Berlin].

[2] P. Berkmann and H.J. Pesch. Abort landing in windshear: optimal control problem with third-order state constraint and varied switching structure. J. of Optimization Theory and Applications, 85, 1995.

[3] J.T. Betts. Practical methods for optimal control using nonlinear programming. Society for Industrial and Applied Mathematics (SIAM), Philadelphia, PA, 2001.

[4] J.F. Bonnans and A. Hermant. Second-order analysis for optimal control problems with pure state constraints and mixed control-state constraints. INRIA Research Report 6199, to appear in Annales de l'Institut Henri Poincaré (C) Analyse Non Linéaire, doi:10.1016/j.anihpc.2007.12.002.

[5] J.F. Bonnans and A. Hermant. Stability and sensitivity analysis for optimal control problems with a first-order state constraint and application to continuation methods. ESAIM Control Optim. Calc. Var. (to appear), E-first DOI: 10.1051/cocv:2008016. 
[6] J.F. Bonnans and A. Hermant. Well-posedness of the shooting algorithm for state constrained optimal control problems with a single constraint and control. SIAM J. on Control and Optimization, 46(4):1398-1430, 2007.

[7] J.F. Bonnans and G. Launay. Large scale direct optimal control applied to a re-entry problem. AIAA J. of Guidance, Control and Dynamics, 21:996-1000, 1998.

[8] B. Bonnard, L. Faubourg, G. Launay, and E. Trélat. Optimal control with state constraints and the space shuttle re-entry problem. J. Dynam. Control Systems, $9(2): 155-199,2003$.

[9] B. Bonnard, L. Faubourg, and E. Trelat. Optimal control of the atmospheric arc of a space shuttle and numerical simulations with multiple-shooting method. Mathematical Models \&3 Methods in Applied Sciences, 15(1):109-140, 2005.

[10] B. Bonnard and E. Trélat. Une approche géométrique du contrôle optimal de l'arc atmosphérique de la navette spatiale. ESAIM: Control, Optimization and Calculus of Variations, 7:179-222, 2002.

[11] R. Bulirsch, F. Montrone, and H. J. Pesch. Abort landing in the presence of windshear as a minimax optimal control problem. II. Multiple shooting and homotopy. J. Optim. Theory Appl., 70(2):223-254, 1991.

[12] A. Hermant. Homotopy algorithm for optimal control problems with a second-order state constraint. INRIA Research Report RR-6626, 2008. http://hal.inria.fr/inria$00316281 / \mathrm{fr} /$.

[13] J. Laurent-Varin, F. Bonnans, N. Bérend, M. Haddou, and C. Talbot. Interior-point approach to trajectory optimization. Journal of Guidance, Control, and Dynamics, 30(5):1228-1238, 2007.

[14] K. Malanowski and H. Maurer. Sensitivity analysis for optimal control problems subject to higher order state constraints. Annals of Operations Research, 101:43-73, 2001. Optimization with data perturbations, II.

[15] H. J. Pesch. A practical guide to the solution of real-life optimal control problems. Control and Cybernetics, 23:7-60, 1994.

[16] H.J. Pesch. Real-time computation of feedback controls for constrained optimal control problems. II. A correction method based on multiple shooting. Optimal Control Appl. Methods, 10(2):147-171, 1989.

\section{Contents}

\begin{tabular}{lll}
\hline & Introduction & 3
\end{tabular}

2 Homotopv algorithm 4

2.1 General framework . . . . . . . . . . . . . . . . . . . . . . 4

2.2 Description of the homotopv algorithm . . . . . . . . . . . . . 5

$\begin{array}{ll}3 \text { Model of atmospheric reentry } & 8\end{array}$

3.1 The optimal control problem . . . . . . . . . . . . . . . . . 8

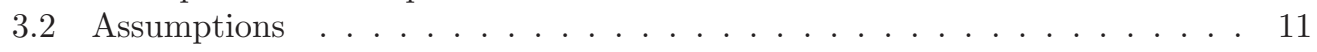

4 Resolution of the unconstrained problem 12

4.1 Resolution of the reduced problem in dimension 3 . . . . . . . . . . . . . . 12

4.2 Resolution of the unconstrained problem . . . . . . . . . . . . . . . . 13 
\begin{tabular}{|lll}
5 & Resolution of the problem with state constraint & 14
\end{tabular} 5.1 Results. . . . . . . . . . . . . . . . . . . . . . . . 14

5.2 Comments on numerical implementation . . . . . . . . . . . . . . 20 


\section{Centre de recherche INRIA Saclay - Île-de-France \\ Parc Orsay Université - ZAC des Vignes \\ 4, rue Jacques Monod - 91893 Orsay Cedex (France)}

Centre de recherche INRIA Bordeaux - Sud Ouest : Domaine Universitaire - 351, cours de la Libération - 33405 Talence Cedex Centre de recherche INRIA Grenoble - Rhône-Alpes : 655, avenue de l'Europe - 38334 Montbonnot Saint-Ismier

Centre de recherche INRIA Lille - Nord Europe : Parc Scientifique de la Haute Borne - 40, avenue Halley - 59650 Villeneuve d'Ascq

Centre de recherche INRIA Nancy - Grand Est : LORIA, Technopôle de Nancy-Brabois - Campus scientifique 615, rue du Jardin Botanique - BP 101 - 54602 Villers-lès-Nancy Cedex

Centre de recherche INRIA Paris - Rocquencourt : Domaine de Voluceau - Rocquencourt - BP 105 - 78153 Le Chesnay Cedex

Centre de recherche INRIA Rennes - Bretagne Atlantique : IRISA, Campus universitaire de Beaulieu - 35042 Rennes Cedex

Centre de recherche INRIA Sophia Antipolis - Méditerranée : 2004, route des Lucioles - BP 93 - 06902 Sophia Antipolis Cedex 